\title{
Moving Horizon Trend Identification Based on Switching Models for Data Driven Decomposition of Fluid Flows
}

\author{
A. Alessandri, P. Bagnerini, M. Gaggero D. Lengani, D. Simoni
}

\begin{abstract}
Modal decomposition is pretty popular in fluid mechanics, especially for data-driven analysis. Dynamic mode decomposition (DMD) allows to identify the modes that describe complex phenomenona such as those physically modelled by the Navier-Stokes equation. The identified modes are associated with residuals, which can be used to detect a meaningful change of regime, e.g., the formation of a vortex. Toward this end, moving horizon estimation (MHE) is applied to identify the trend of the norm of the residuals that result from the application of DMD for the purpose to automatically classify the time evolution of fluid flows. The trend dynamics is modelled as a switching nonlinear system and hence an MHE problem is solved in such a way to monitor the time behavior of the fluid and quickly identify changes of regime. The stability of the estimation error given by MHE is proved. The combination of DMD and MHE provide successful results as shown by processing experimental datasets of the velocity field of fluid flows obtained by a particle image velocimetry.
\end{abstract}

\section{INTRODUCTION}

The motion of a fluid flow is described by the NavierStokes equation. This equation can be directly solved by means of advanced computational model such as the socalled direct numerical simulation (DNS), which are nowadays adopted even for complex geometries. This solution leads to the generation of large amount of data from which it is often difficult to directly extract useful information. The same issues arise with the experimental measurements of the velocity field of fluid flows such as those obtained by a particle image velocimetry (PIV).

The large amount of information available by DNS and PIV need to be properly reduced to simple models in order to be adopted by designers for the purpose of analysis as well as for devising some control setup of the fluid flow. Among the recent post-processing tools, the Dynamic Mode Decomposition (DMD) is one of the most promising to reduce the computational difficulties to deal with such complex systems [1]. This technique isolates the main dynamics of the system under investigation by providing a simpler representation of the dominant structures (the modes of the decompositions) and their temporal evolution. Namely, the DMD extracts the dominant dynamics through the linear transformation best mapping (in a least square sense) of successive ensembles of snapshots. Based on linear system theory, this technique identifies the instability waves [2] and

A. Alessandri P. Bagnerini, D. Lengani, and D. Simoni are with the University of Genoa, DIME, Genova, Italy (e-mail: alessandri@dime.unige.it, bagnerini@dime.unige.it, davide.lengani@edu.unige.it, daniele.simoni@unige.it).

M. Gaggero is with the National Research Council of Italy, Genova, Italy (e-mail: mauro.gaggero@cnr.it). provides the best linear approximation to their dynamics [1] Thus, the peculiar information raised by the DMD concerns with the identification of modes, their frequencies, and their growth (or decay) rates [1]-[3]. However, the quality of the results provided by such techniques depends on the complexity of the dynamics of the fluid flow system. In particular, unsteady flows that are characterized by a full spectrum in the frequency domain require a careful analysis.

The evolution of fluid flows is described by the NavierStokes equation, which is still today a challenge to be analyzed and poses also additional issues because of the necessity to deal with large amount of data. However, the use of such equation may be really successful in improving the quality of the results (see, e.g., [4]). DMD and proper orthogonal decomposition (POD) [5], [6] tools allow for reducing the degrees-of-freedom of a complex system (the boundary layer in our case), thus the order of the problem to be inspected. Moreover, they isolate the prevailing dynamics of the fluid flow under investigation by providing a representation of the dominant structures (the modes of the decompositions) and their temporal and spatial evolution.

In this paper, we investigate the use of moving horizon estimation (MHE) in order to identify the trend of the norm of the residuals that result from the linear mapping obtained by using DMD. The identification of such a trend allows to automatically detect the switching between different regimes in a boundary layer flow, and consequently to extract modes, frequencies, and growth rates in an accurate way. The proposed method is used to treat experimental data obtained by time resolved particle image velocimetry (TR-PIV) with the aim to understand the transition processes of the boundary layers growing along turbomachinery blades.

The identification of a trend may be cast as a constrained estimation problem and MHE is particularly well-suited to performing estimation under constraints in real time. The literature on MHE is vast and concerns linear systems [7] [10], nonlinear systems [11]-[13], switching systems [14], [15], and uncertain systems [16]-[19]. Here we focus on identification of different fluid flow conditions, where, to get a reliable estimate, we need to account for constraints on the state estimates as well as on the occurrence of switching. This may be important, for instance, if the estimate of the specific flow conditions is used to set some control setup.

We will adopt the following notation. The symbol $(x, y)$, where $x$ and $y$ are column vectors, stands for $\left[x^{\top}, y^{\top}\right]^{\top}$. Given a generic matrix $M,|M|:=\left(\lambda_{\max }\left(M^{\top} M\right)\right)^{1 / 2}=$ $\left(\lambda_{\max }\left(M M^{\top}\right)\right)^{1 / 2}$. Accordingly, in the special case of a 
vector $v,|v|:=\left(v^{\top} v\right)^{1 / 2}$ denotes its Euclidean norm. The Frobenius norm of a matrix $M$ is $|M|_{F}:=\sqrt{\operatorname{tr}\left(M^{\top} M\right)}$. Given a complex matrix $C, C^{*}$ denotes its Hermitian.

\section{ESTIMATION OF A TREND DYNAMICS}

Consider the problem of estimating the trend of a nonnegative sequence of measurements of a random process that may be either decreasing or increasing (see Fig. 1).

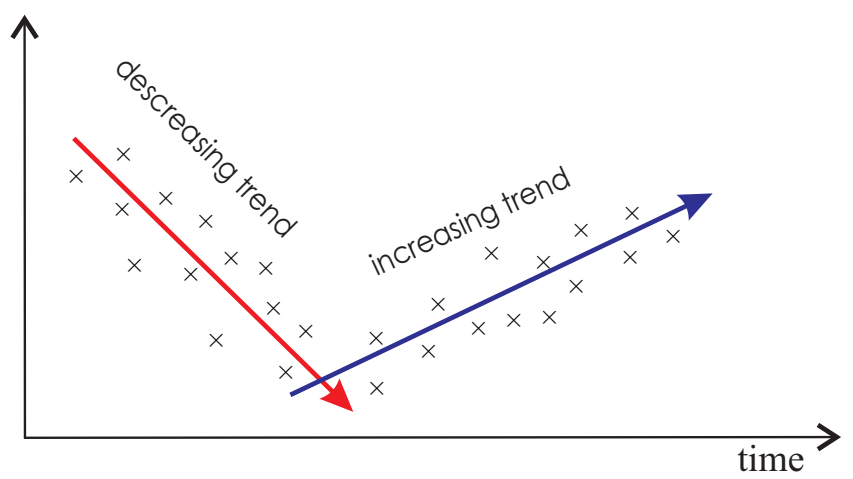

Fig. 1. Sketch of a sequence with a turning point from decreasing to increasing trend.

A simple dynamics with a either decreasing or increasing trend after a turning point can be modelled by the 2-mode switching system

$$
\begin{aligned}
& \text { mode } 1\left\{\begin{array}{l}
x_{1}(t+1)=x_{2}(t) x_{1}(t) \\
x_{2}(t+1)=\alpha_{1} \in(0,1) \\
y(t)=x_{1}(t)
\end{array}\right. \\
& \text { mode } 2\left\{\begin{array}{l}
x_{1}(t+1)=x_{2}(t) x_{1}(t) \\
x_{2}(t+1)=\alpha_{2} \in(1,+\infty) \\
y(t)=x_{1}(t)
\end{array}\right.
\end{aligned}
$$

at time instants $t=0,1, \ldots$ and with $\alpha_{1}$ and $\alpha_{2}$ unknown. The problem of identifying the trend reduces to find constrained estimates of $\alpha_{1}$ and $\alpha_{2}$ together with a decision on the turning point, which is of course unknown. Such estimates may be affected by disturbances on the measures, thus a MHE estimation approach may be well-suited to performing this task for its robustness and the possibility to account for the intrinsic nonlinear and switching dynamics.

Consider the switching discrete-time system

$$
\begin{aligned}
x_{t+1} & =f_{\lambda_{t}}\left(x_{t}, u_{t}\right) \\
y_{t} & =h_{\lambda_{t}}\left(x_{t}, u_{t}\right)
\end{aligned}
$$

where $t=0,1, \ldots, x_{t} \in X \subset \mathbb{R}^{n}$ is the continuous state vector, $\lambda_{t} \in\{1, \ldots, q\}$ is the discrete state or system mode, $u_{t} \in U \subset \mathbb{R}^{p}$ is the control vector, and $y_{t} \in \mathbb{R}^{m}$ is the output vector. The functions $f_{i}: \mathbb{R}^{n} \times \mathbb{R}^{p} \rightarrow \mathbb{R}^{n}$ and $h_{i}$ : $\mathbb{R}^{n} \times \mathbb{R}^{p} \rightarrow \mathbb{R}^{m}$ are smooth. The set $X$ and $U$ are assumed to be compact. System (2) is subject to the equality constraint

$$
g_{\lambda_{t}}\left(x_{t}, u_{t}\right) \leq 0, t=0,1, \ldots
$$

where each $g_{i}: \mathbb{R}^{n} \times \mathbb{R}^{p} \rightarrow \mathbb{R}^{q}$ is smooth. Since the goal consists in devising a moving-horizon estimator that relies on the most recent batch of input and output data, let us define $y_{t-N}^{t}:=\left(y_{t}, y_{t-1}, \ldots, y_{t-N}\right)$ and $u_{t-N}^{t}:=\left(u_{t}, u_{t-1}, \ldots\right.$, $\left.u_{t-N}\right)$. Concerning the discrete state, not all the possible combinations of discrete states may in general occur; therefore, after defining $\lambda_{t-N}^{t}:=\left(\lambda_{t}, \lambda_{t-1}, \ldots, \lambda_{t-N}\right)$ a generic switching pattern from time $t-N$ to $t$, let us denote by $\Lambda_{0}^{N}$ the set of the admissible switching patterns. Moreover, let

$$
\begin{aligned}
& H\left(x_{t-N}, u_{t-N}^{t}, \lambda_{t-N}^{t}\right) \\
& :=\left(\begin{array}{c}
h_{t}^{u, \lambda} \circ f_{t-1}^{u, \lambda} \circ f_{t-2}^{u, \lambda} \circ \cdots \circ f_{t-N}^{u, \lambda}\left(x_{t-N}\right) \\
h_{t-1}^{u, \lambda} \circ f_{t-2}^{u, \lambda} \cdots \circ f_{t-N}^{u, \lambda}\left(x_{t-N}\right) \\
\vdots \\
h_{t-N+1}^{u, \lambda} \circ f_{t-N}^{u, \lambda}\left(x_{t-N}\right) \\
h_{t-N}^{u, \lambda}\left(x_{t-N}\right)
\end{array}\right) \in \mathbb{R}^{(N+1) m}
\end{aligned}
$$

where $f_{t}^{u, \lambda}\left(x_{t}\right):=f_{\lambda_{t}}\left(x_{t}, u_{t}\right)$ and $h_{t}^{u, \lambda}\left(x_{t}\right):=h_{\lambda_{t}}\left(x_{t}, u_{t}\right)$ for the sake of brevity. Thus, $y_{t-N}^{t}=H\left(x_{t-N}, u_{t-N}^{t}, \lambda_{t-N}^{t}\right)$.

Assumption 1 (Uniform continous-discrete observability): There exists $\delta>0$

$$
\delta\left|x^{\prime}-x^{\prime \prime}\right| \leq\left|H\left(x^{\prime}, u_{t-N}^{t}, \lambda_{t-N}^{t}\right)-H\left(x^{\prime \prime}, u_{t-N}^{t}, \lambda_{t-N}^{t}\right)\right|
$$

for all $u_{t-N}^{t} \in U^{N+1}$ and $\lambda_{t-N}^{t} \in \Lambda_{0}^{N}$.

An MHE strategy for (2) relies on the information obtained in the recent past by computing a state estimate that minimizes a least-squares cost

$$
\begin{aligned}
& J_{t}\left(\hat{x}_{t-N}^{t}, \hat{\lambda}_{t-N}^{t}\right)=\mu\left|\hat{x}_{t-N}-\bar{x}_{t-N}\right|^{2}+\sum_{i=t-N}^{t} \mid y_{i} \\
& -\left.h_{\hat{\lambda}_{i}}\left(\hat{x}_{i}, u_{i}\right)\right|^{2}=\mu\left|\hat{x}_{t-N}-\bar{x}_{t-N}\right|^{2} \\
& +\left|y_{t-N}^{t}-H\left(\hat{x}_{t-N}, u_{t-N}^{t}, \hat{\lambda}_{t-N}^{t}\right)\right|^{2}
\end{aligned}
$$

with $\mu>0$ and where $\hat{x}_{t-N}^{t}$ and $\hat{\lambda}_{t-N}^{t}$ are the estimates of $x_{t-N}^{t}$ and $\lambda_{t-N}^{t}$, respectively.

Problem 1: At each $t$ solve the problem $\min J_{t}\left(\hat{x}_{t-N}^{t}, \hat{\lambda}_{t-N}^{t}\right)$ w.r.t. $\hat{x}_{t-N}^{t} \in X^{N+1}, \hat{\lambda}_{t-N}^{t} \in \Lambda_{0}^{N}$ s.t.

$\hat{x}_{i+1}=f_{\hat{\lambda}_{i}}\left(\hat{x}_{i}, u_{i}\right), i=t-N, \ldots, t-1$

$g_{\hat{\lambda}_{i}}\left(\hat{x}_{i}, u_{i}\right) \leq 0, i=t-N, \ldots, t$.

At each time $t$ we solve Problem 1 by obtaining $\hat{x}_{t-N}^{\mathrm{o}}$ and $\hat{\lambda}_{t-N}^{o}$ to compute $\bar{x}_{t-N+1}=f_{\hat{\lambda}_{t-N}^{o}}\left(\hat{x}_{t-N}^{o}, u_{t-N}\right)$ as a prediction of $x_{t-N+1}$. Based on such a prediction, we solve again Problem 1 at time $t+1$ and so on, as sketched in Fig. 2.

Example 1: Consider again (1), which can equivalently expressed as follows:

$$
\begin{aligned}
& x_{t+1}=f_{\lambda_{t}}\left(x_{t}\right) \\
& y_{t}=C x_{t}
\end{aligned}
$$

where $C=\left(\begin{array}{ll}1 & 0\end{array}\right)$ and

$$
f_{\lambda_{t}}\left(x_{t}\right):=\left[\begin{array}{c}
x_{1, t} x_{2, t} \\
x_{2, t}
\end{array}\right]
$$




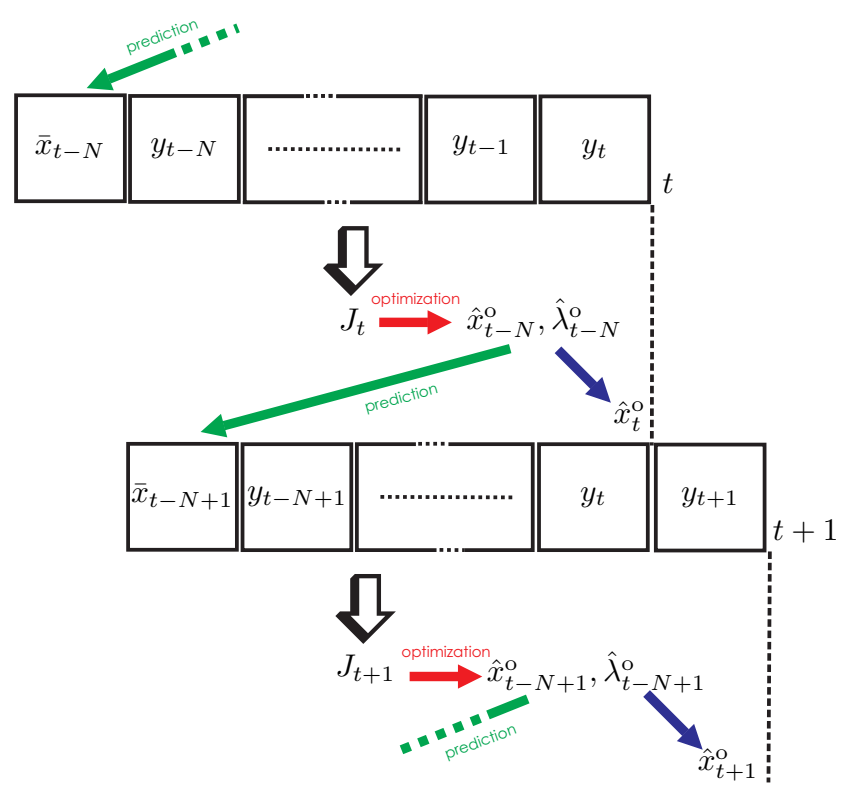

Fig. 2. MHE strategy from time $t$ to $t+1$.

with $x_{t}=\left(x_{1, t}, x_{2, t}\right)$ and $x_{2, t}=\alpha_{i}$ if $\lambda_{t}=i, i=1,2$. Thus, in case of $N=1$ (i.e., a moving with the last two measures), we deal with $y_{t-1}^{t}$ and hence, to simplify the notation, let us refer to

$$
y_{0}^{1}=H\left(x_{0}, \lambda_{0}^{1}\right)=\left[\begin{array}{c}
x_{1,0} x_{2,0} \\
x_{2,0}
\end{array}\right] \text {. }
$$

The simultaneous distinguishability of continuous and discrete states corresponds to the satisfaction of the following condition

$$
H\left(\bar{x}_{0}, \bar{\lambda}_{0}^{1}\right)=H\left(\tilde{x}_{0}, \tilde{\lambda}_{0}^{1}\right) \Rightarrow \bar{x}_{0}=\tilde{x}_{0} \text { and } \bar{\lambda}_{1}=\tilde{\lambda}_{1} .
$$

Such a condition is trivially satisfied for $\tilde{x}_{1,0}, \bar{x}_{1,0} \neq 0$, which is necessary for the switching patterns $(1, \star)$ and $(2, \star)$ to be distinguishable (the symbol $\star$ means the condition is independent from the initial mode). Moreover, we get

$$
\frac{\partial H\left(x_{0}, \lambda_{0}^{1}\right)}{\partial x_{0}}=\left[\begin{array}{cc}
x_{2,0} & x_{1,0} \\
1 & 0
\end{array}\right] .
$$

Since for $x_{1,0} \neq 0$ we have that the rank of (8) is maximum and (7) is satisfied, we can apply [20][Proposition 10, p. 1036] and conclude that (4) and hence Assumption 1 hold over compact sets that do not intersect the set $\left\{x \in \mathbb{R}^{2}\right.$ : $C x=0\}$.

To proceed with the stability results, we need to assume the following.

Assumption 2: The functions $f$ and $h$ are of class $C^{2}$ and

$$
\left|f_{\lambda}(\bar{x}, u)-f_{\lambda}(\tilde{x}, u)\right| \leq k_{f}|\bar{x}-\tilde{x}|, \forall \bar{x}, \tilde{x} \in X
$$

for some $k_{f}>0$ and independently of $u \in U$ and $\lambda \in$ $\{1, \ldots, q\}$.

The solution of Problem 1 provides a stable estimation error.
Theorem 1: If $\mu$ is chosen such that

$$
\frac{4 \mu k_{f}^{2}}{\mu+2 \delta^{2}}<1
$$

the estimation error $e_{t}:=x_{t}-\hat{x}_{t}$ is exponentially stable to zero.

Proof. The proof is based on the use of lower and upper bounds on the cost (5) in line with previous results reported in the literature [12], [15]. The lower bound is derived by using Assumption 1. The cost is upper bounded by exploiting the definition of prediction backward, i.e., using the relation $\bar{x}_{t-N+1}=f_{\hat{\lambda}_{t-N}^{o}}\left(\hat{x}_{t-N}^{\mathrm{o}}, u_{t-N}\right)$.

If the dynamic and measurement equations are affected by bounded, additive noises, the estimation error is exponentially bounded ${ }^{1}$.

Theorem 2: Suppose that

$$
\begin{aligned}
x_{t+1} & =f_{\lambda_{t}}\left(x_{t}, u_{t}\right)+w_{t} \\
y_{t} & =h_{\lambda_{t}}\left(x_{t}, u_{t}\right)+v_{t}
\end{aligned}
$$

where $t=0,1, \ldots$ and bounded disturbances $w_{t}$ and $v_{t}$. Let $\mu$ chosen such that

$$
\frac{8 \mu k_{f}^{2}}{\mu+\delta^{2}}<1
$$

Then the estimation error is exponentially bounded.

Proof. It is along the line of the proof of [11, Theorem 1, p. 1758]. As compared with the stability conditions of Theorem 1, (11) turns out to be more restrictive since the bounds derived in the noise-free setting are tighter for avoiding the passages required to account for the system and measurement disturbances.

Theorems 1 and 2 enable to perform trend estimation with stability guarantees by using (1) according to the proposed approach and thanks to the satisfaction of the observability condition as detailed in Example 1.

\section{DynAmic Mode Decomposition}

The Navier-Stokes equation is pretty popular in fluid mechanics since it is able to describe the full evolution of a fluid flow system. Such system can be highly complex and it is described by a big amount of data that requires a proper reduction. DMD is an analysis technique recently proposed in [1] that is applicable for numerical as well as experimental flow field data. Given a series of data with the temporal or spatial evolution of a fluid flow, the DMD provides the best linear transformation (in the least square sense) mapping a flow field snapshot sequence into a successive one. This sequence may be over space or time. For example, let us suppose to have at disposal a dataset of a two-dimensional velocity field made up by $M$ repeated snapshots. If we define $V_{1}$ as the collection of snapshots 1 to $M$ and $V_{2}$ a similar

\footnotetext{
${ }^{1}$ A sequence $v_{t} \in \mathbb{R}^{n}$ is said to be exponentially bounded if there exists $a \in(0,1)$ and $b>0$ such that $\left|v_{t}\right| \leq\left|v_{0}\right| a^{t}+b, t=0,1, \ldots$ [11]
} 
collection but with the snapshots from 2 to $M+1$, the DMD results from the solution of the minimization problem

$$
\min _{S \in \mathcal{S}}\left|V_{2}-V_{1} S\right|_{F}^{2}
$$

where the unknown $S$ is a transformation matrix of appropriate dimension and in canonical form given by

$$
S=\left(\begin{array}{ccccc}
0 & 0 & \cdots & \cdots & a_{1} \\
1 & 0 & \cdots & \cdots & a_{2} \\
& \ddots & \ddots & & \vdots \\
& & 1 & 0 & a_{M-1} \\
& & 0 & 1 & a_{M}
\end{array}\right), a \in \mathbb{R}^{M}
$$

and $\mathcal{S}$ denotes the set of all real matrices having the structure above.

Thus, the DMD allows to find a linear dynamics that approximate the underlying process and can provide additional information through the analysis of the eigenvalues and the eigenvectors of $S$. An efficient algorithm for the decomposition of the matrix $S$ has been proposed in [1] by projecting the matrix into the sub-space spanned by the POD modes of $V_{1}$. The DMD modes and eigenvalue are then obtained from a full rank matrix $\tilde{S}$ related via a similarity transformation to the matrix $S$. The DMD algorithm is composed of the following steps:

1) compute the singular value decomposition of $V_{1}$, i.e., two unitary $U$ and $W$ and a diagonal matrix $\Sigma$ s.t. $V_{1}=U \Sigma W^{*}$

2) compute the projection of the matrix $S$ on the POD modes, i.e., $\tilde{S}=U^{*} V_{2} W \Sigma^{-1}$;

3) construct the matrices of the eigenvectors and eigenvalues of the matrix $\tilde{S}$, i.e., $Y$ and $D$ (diagonal);

4) construct the matrix of the DMD modes $\Theta=U Y$;

5) construct the diagonal matrix $P$ of the logarithms of the DMD eigenvalues of $\tilde{S}$, i.e., $P:=\log (D) /(2 \pi \Delta t)$, where $\Delta t$ is the time between two consecutive snapshots.

Using DMD, coherent structures in the flow can be easily localized by a check on modes and eigenvalues [21]. The frequency information is given by the imaginary part of the DMD eigenvalue, while the real part of the eigenvalue provides the growth rate of the dynamic structure identified by the corresponding DMD mode. Positive values of the real part of the DMD eigenvalues indicate growing structures. Thus, the dominant dynamics leading to the generation of unstable waves within the flow can be captured and highlighted. Particularly, the transition of the boundary layer is identified as the region where positive growth rate are measured and its characteristic frequency can be computed in this way. Otherwise, stable regime such as the laminar state (prior to the transition) and the turbulent one (after transition) have just negative or null growth rate. Therefore, in order to correctly compute the frequencies and the growth rate, it is necessary to detect the spatial positions, where the switching between different regimes takes place. This corresponds to identify the snapshot of the dataset at which the switching occurs. In order to minimize the amount of

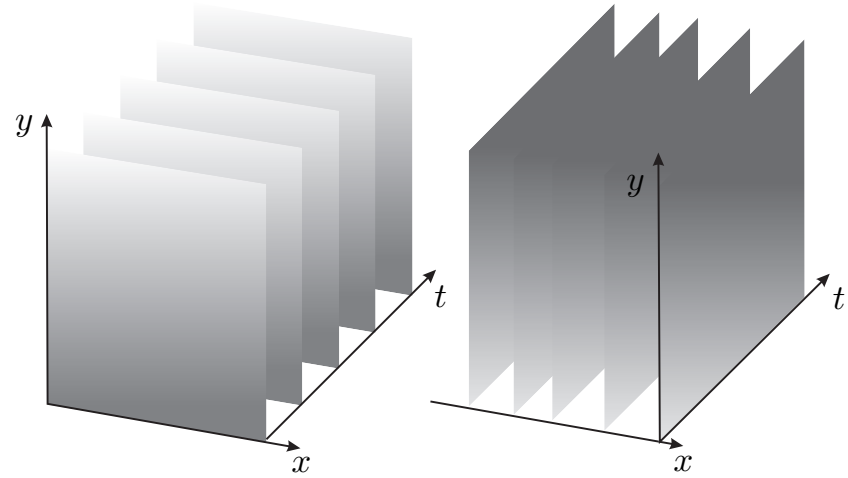

Fig. 3. Arrangements of the dataset for temporal (left) or spatial (right) analysis.

analyzed data, the temporal interval has been chosen in order to represent about 10 cycles of the vortical structures that drives transition. However, it has been shown [21] that the wall-normal velocity component is more representative of the switching of the two regimes since before transition starts it is almost null, hence it is used to compute the residual of DMD algorithm. Both components are instead used to compute frequencies and growth rate, since the whole information is needed to obtain a great accuracy.

To detect the switching, we have developed the following procedure based on DMD algorithm for a dataset composed of $M$ snapshots. Let $k$ be a candidate for the spatial position of the switching, corresponding to a snapshot (or column) of the dataset. We scroll the columns of the dataset from $k=k_{0}, \cdots, M$ and we apply the DMD algorithm to a subset of the dataset formed by the first $k$ columns. The DMD algorithm is applied to such a subset, having defined $V_{1, k}$ constituted by the snapshots 1 to $k-1$, and $V_{2, k}$ by the snapshots 2 to $k$, computes the solution of the minimization problem (12). The corresponding residual matrix with $k$ columns is $R_{k}:=V_{2, k}-V_{1, k} S_{k}^{\text {o, }}$, where $S_{k}^{\circ}=\operatorname{argmin}_{S \in \mathcal{S}}\left|V_{2, k}-V_{1, k} S\right|_{F}^{2}$. To identify the change of regime, we will employ the cumulative average $r_{k}$ of the Euclidean norm of the columns of $R_{k}$, i.e.,

$$
r_{k}:=\frac{\left|\left(R_{1}, R_{2}, \ldots, R_{k}\right)\right|}{k}
$$

at each $k$. Therefore, we will compute $r_{k}$ for $k=k_{0}, \cdots, M$ and apply the approach of Section II to estimate the trend and detect the change of regime in the fluid flow. Note that, the index $k$ may refer also to space rather than to time since in practice one may apply DMD can be applied in either time or space. Moreover, from the next section and in spite of the notation adopted so far, we will refer to $x$ and $y$ as the spatial coordinates to denote the axes of the velocity field. Each column of the dataset corresponds to a snapshot made of a collection of measurements of the velocities of the flow over $y$ and time. Thus, in the following the index $k$ will denote space in the $x$ coordinate. 


\section{EXPERIMENTAL INVESTIGATION}

The experiments have been performed in the open-circuit low-speed wind tunnel of the Aerodynamics and Turbomachinery Laboratory of the University of Genova. Fig. 4 shows the experimental setup that consists of a thick flat plate. The flat part of the plate including the leading edge is 200 $\mathrm{mm}$ long and $300 \mathrm{~mm}$ wide. The plate has been installed between two contoured walls producing an adverse pressure gradient. The boundary layer developing along the rear part of the plate was surveyed by means of a DANTEC TR-PIV system. The measuring domain extends from $x / c=0.315$ to $x / c=0.9$ (the test section throat is located at $x / c=$ 0.285). A dataset of 3100 instantaneous velocity fields has been acquired at a sampling rate of $3100 \mathrm{~Hz}$. The elevated frequency resolution and the long sampling period (1 s) of the present dataset allow us to follow the dynamics leading to the generation of the large scale coherent structures and hence to transition.

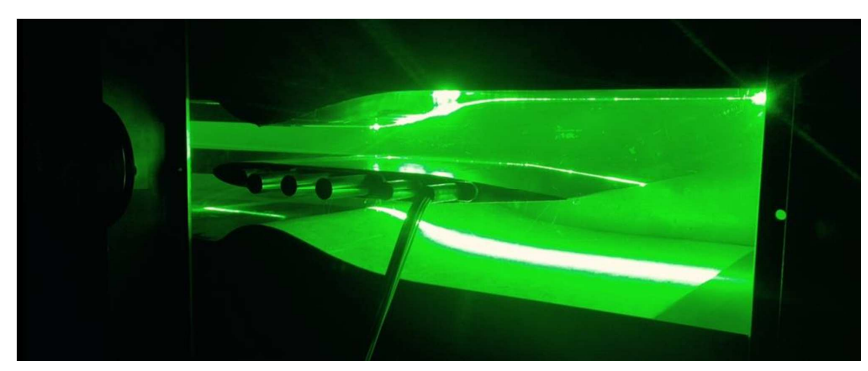

Fig. 4. Test section and experimental apparatus.

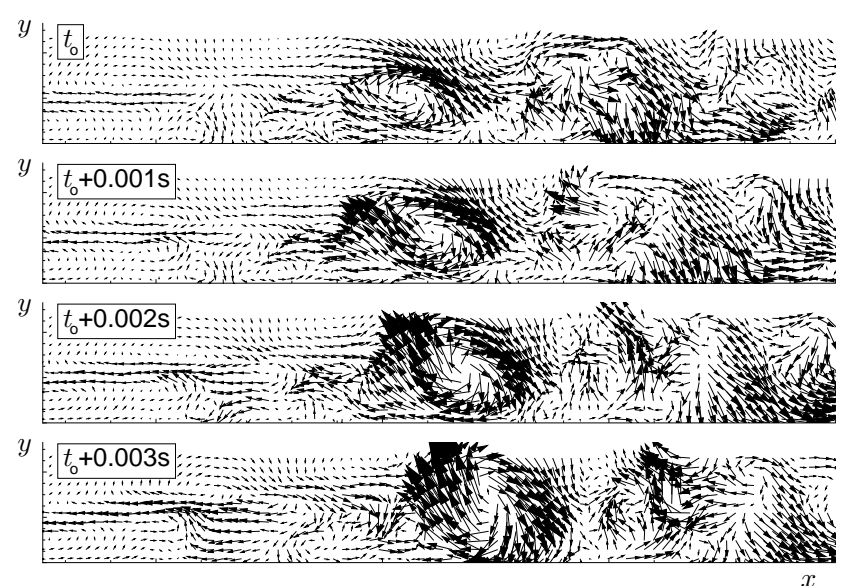

Fig. 5. Velocity field of instantaneous flow fields at different time instants.

The PIV instrumentation is constituted by a dual-cavity Nd:YLF pulsed laser Litron LDY 300 (energy $2 \times 30 \mathrm{~mJ}$ per pulse at $1000 \mathrm{~Hz}$ repetition rate, $527 \mathrm{~nm}$ wavelength). The optical system forms a light sheet of $1 \mathrm{~mm}$ thickness. The light scattered by the seeding particles is recorded on a high sensitive SpeedSense M340 digital camera with a cooled $2560 \times 1600$ pixels CMOS matrix. The camera frame rate has been set to $3100 \mathrm{~Hz}$ reducing the field of view of the camera to $2560 \times 200$ and operating it in double frame mode.
The spatial resolution of the measurement grid corresponds to $0.43 \times 0.43 \mathrm{~mm}^{2}$. This spatial resolution allows to solve the large scale structures that trigger the switch between the different flow regimes whose analysis is the main target of the present work. More details concerning the measurement uncertainty and flow conditions are available in [21].

Fig. 5 shows a series of the acquired instantaneous flow field, showing one out of three of the acquired images. For visualization purposes, the spatial resolution has been also reduced plotting one every 2 measurement points in both directions. The velocity field shown in the pictures has been obtained subtracting the mean value in order to better visualize the coherent structures. It can be noticed that the DMD is instead computed without subtracting the time mean velocity otherwise it would be equal to a classical Fourier analysis [3].

The four snapshots of Fig. 5 highlight the temporal and spatial evolution of a vortical structure that can be observed in the mid of the first frame. This structure is propagating in space and time since from the first frame to the fourth it can be seen moving in the downstream direction. While moving, the vortical structure intensity (that can be quantified by the vector length) is also increasing. Hence, from a qualitative point of view, the visual inspection of the instantaneous flow field could be used to identify a change in regime of the boundary layer flow. Otherwise, the analysis of the residuals of the DMD is here adopted to provide a quantitative analysis of the switching of these regimes.

\section{NUMERICAL RESULTS}

To identify the change of regime, consider (1) with output $r_{k}$, i.e.,

$$
\begin{aligned}
& \text { mode } 1\left\{\begin{array}{l}
\xi_{1}(k+1)=\xi_{2}(k) \xi_{1}(k) \\
\xi_{2}(k+1)=\alpha_{1} \in(0,1) \\
r_{k}=\xi_{1}(k)
\end{array}\right. \\
& \text { mode } 2\left\{\begin{array}{l}
\xi_{1}(k+1)=\xi_{2}(k) \xi_{1}(k) \\
\xi_{2}(k+1)=\alpha_{2} \in(1,+\infty) \\
r_{k}=\xi_{1}(k)
\end{array}\right.
\end{aligned}
$$

where $r_{k}$ is computed according to DMD procedure presented in Section III is applied from $k=k_{0}$ with $k_{0}=20$.

We have solved the MHE problem (6) for the above system model by using the cost (5) with $\mu=0.001$ and different horizon lengths $N$, i.e., $N=1, N=3, N=5$, and $N=7$. We have performed the minimization of (5) in Matlab on a PC equipped with a $2.5 \mathrm{GHz}$ Intel Xeon CPU and $16 \mathrm{~GB}$ of RAM by using the fmincon routine with constraints given by the positivity of the variables and the kind of trend.

Fig. 6 depicts the positions of the poles at different regime, i.e., in correspondence of stable and unstable behavior of the fluid flow. Fig. 7 reports the results we have obtained by using the proposed MHE approach. The subplot (a) shows the various type of residuals, i.e., "original," filtrated with a mobile average with sliding window of length 10 , and "estimated" by the MHE with different horizons. The subplot (b) sketches the values of the state variable $\xi_{2}$. The subplot (c) reports the estimated modes of (1). The modes 

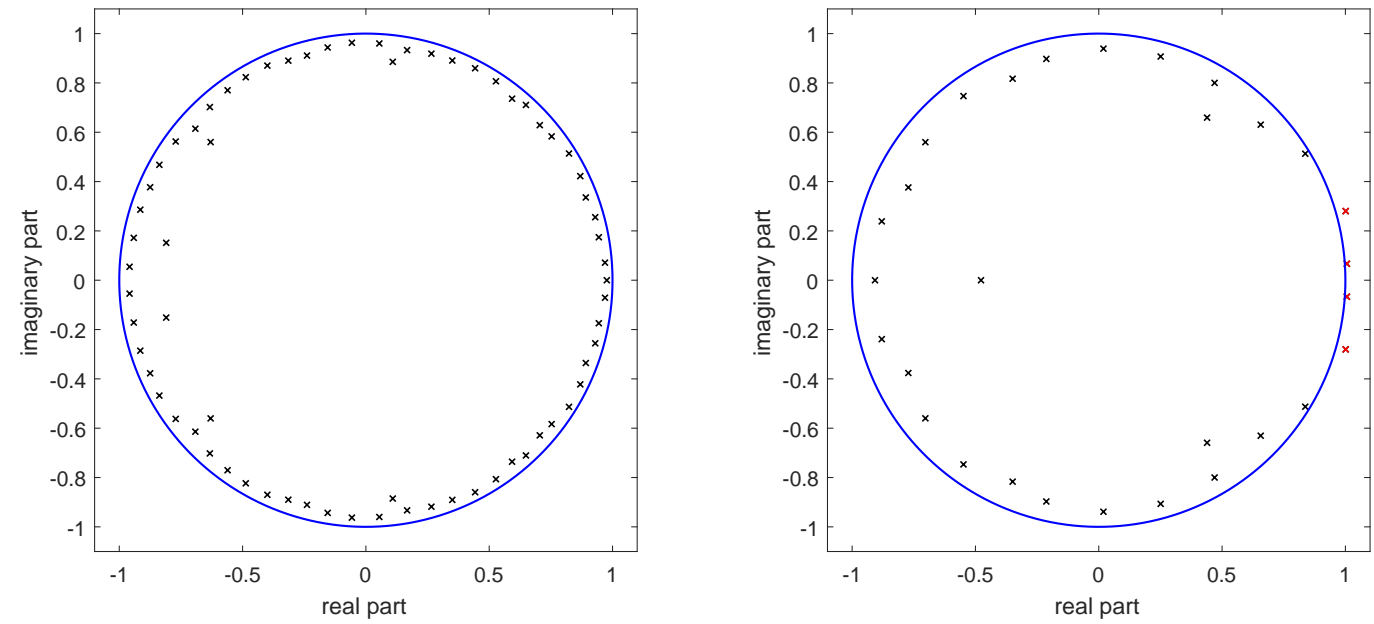

Fig. 6. Positions of the poles resulting from the DMD at stable and unstable regimes.

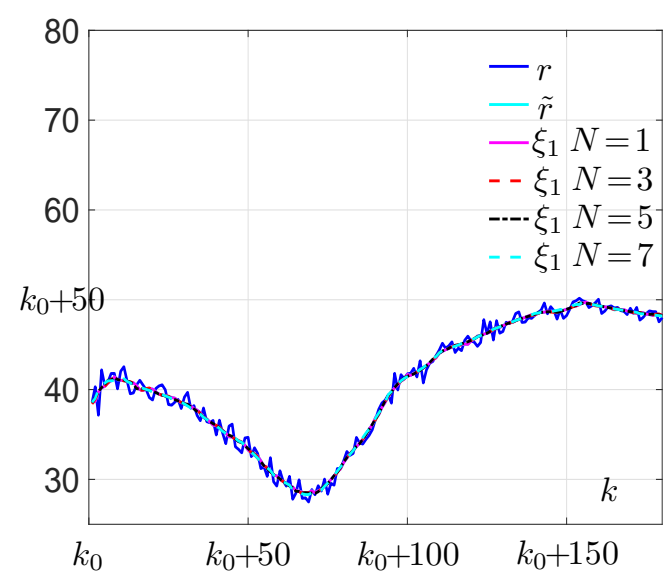

(a)

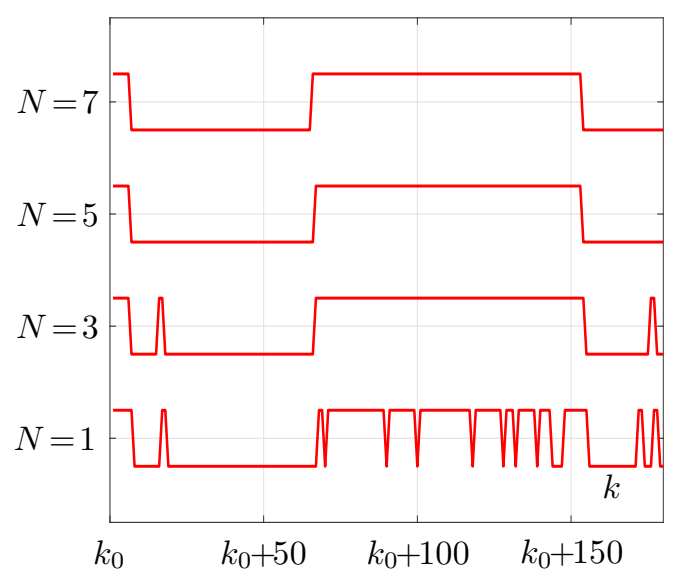

(c)

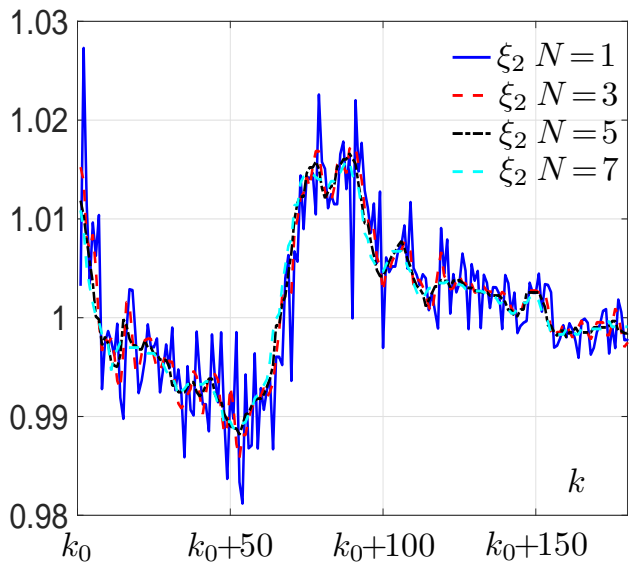

(b)

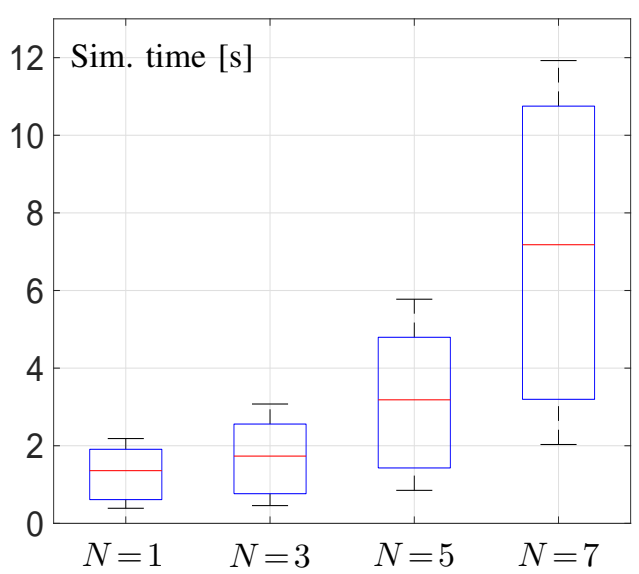

(d)

Fig. 7. Experimental results obtained for different horizons $N$.

are pictorially shown as step signals, where the modes 1 and 2 are represented by the low and high values of the step, respectively. Lastly, the subplot (d) illutrates the boxplots of the processing times required to solve an instance of the MHE problem (6).

The MHE approach is able to correctly identify the 
decreasing/increasing trend of the residuals for large values of $N$. More specifically, the larger is $N$, the better are the performances. On the contrary, the use of short horizons $N$ makes the proposed approach more sensitive to noises. This is particularly evident in the plot of the state variable $\xi_{2}$, which is very noisy for $N=1$ and much better for larger values of $N$. The estimation accuracy of the state variable $\xi_{1}$ is quite insensitive to the horizon length since this variable is directly measured. Concerning the computational effort, as expected, the greater the horizon $N$, the larger the simulation times, as the solution of (6) is more difficult with the growth of $N$. The reduction of the computational time may be the topic of future investigation in such a way to make it possible to apply the proposed approach in real time.

\section{CONCLUSIONS}

In this paper, we have presented a novel approach to the automatic identification of the regime of turbulent flows by using (i) dynamic mode decomposition, (ii) a switching, nonlinear trend model, and (iii) moving horizon estimation. The combination of them allows for a reliable estimation of the complex phenomena occurring in a fluid flow, as shown by applying the proposed approach on an experimental dataset obtained by a PIV.

The successful results we have illustrated indicate various directions of improvement. For example, a future goal may be that of developing a more complex model of trend able to identify three regimes instead of two, which correspond to 1) a stable laminar regime with low velocity fluctuations, 2) a new regime with the amplification of the disturbances, and 3) a turbulent regime that is stable but characterized by large velocity fluctuations.

Another topic of future investigation will be the reduction of the computational effort of the estimation in such a way to detect the state of the flow more quickly for the purpose of control. Toward this, the use of fast MHE techniques [13] will be addressed in such a way to perform the optimization within a time constraint that allows to apply a stabilizing feedback to the fluid flow.

\section{REFERENCES}

[1] P. Schmid, "Dynamic mode decomposition of numerical and experimental data," Journal of Fluid Mechanics, vol. 656, pp. 5-28, 2010.

[2] S. Sarmast, R. Dadfar, R. F. Mikkelsen, P. Schlatter, S. Ivanell, J. Sorensen, , and D. S. Henningson, "Mutual inductance instability of the tip vortices behind a wind turbine," J. Fluid Mech., vol. 755, pp. 705-731, 2014.

[3] K. Chen, J. Tu, and C. Rowley, "Variants of dynamic mode decomposition: boundary condition, Koopman, and Fourier analyses," Journal of Nonlinear Science, vol. 22, no. 6, pp. 887-915, 2012.

[4] S. Zhuk, T. Tchrakian, A. Akhriev, S. Lu, and H. Hamann, "Dynamic cloud motion forecasting from satellite images," in Proc. 56th Annual Conference on Decision and Control, Melbourne, Australia, 2015, pp. 3107-3112.

[5] J. Borggaard and A. Lattimer, "POD models for positive fields in advection-diffusion-reaction equations," in 2017 American Control Conference (ACC), 2017, pp. 3797-3802.

[6] S. Sahyoun and S. M. Djouadi, "Orthogonal locality preserving model reduction and flow separation control for incompressible Navier Stokes equations," in 2017 American Control Conference (ACC), 2017, pp. 3803-3808.
[7] C. Rao, J. Rawlings, and J. Lee, "Constrained linear estimation-a moving horizon approach," Automatica, vol. 37, no. 10, pp. 1619$1628,2001$.

[8] C. Rao, J. Rawlings, and D. Mayne, "Constrained state estimation for nonlinear discrete-time systems: stability and moving horizon approximations," IEEE Trans. Automat. Control, vol. 48, no. 2, pp. 246-257, 2003.

[9] A. Alessandri, M. Baglietto, and G. Battistelli, "Receding-horizon estimation for discrete-time linear systems," IEEE Trans. Automat. Control, vol. 48, no. 3, pp. 473-478, 2003.

[10] A. Voelker, K. Kouramas, and E. Pistikopoulos, "Moving horizon estimation: Error dynamics and bounding error sets for robust control," Automatica, vol. 49, no. 4, pp. 943 - 948, 2013.

[11] A. Alessandri, M. Baglietto, and G. Battistelli, "Moving-horizon state estimation for nonlinear discrete-time systems: New stability results and approximation schemes," Automatica, vol. 44, no. 7, pp. 17531765,2008

[12] A. Alessandri, M. Baglietto, G. Battistelli, and M. Gaggero, "Movinghorizon state estimation for nonlinear systems using neural networks," IEEE Trans. Neural Networks, vol. 22, no. 5, pp. 768-780, 2011.

[13] A. Alessandri and M. Gaggero, "Fast moving horizon state estimation for discrete-time systems using single and multi iteration descent methods," IEEE Trans. Automat. Control, vol. 62, no. 9, pp. 44994511, 2017.

[14] A. Alessandri, M. Baglietto, and G. Battistelli, "Receding-horizon estimation for switching discrete-time linear systems," IEEE Trans. Automat. Control, vol. 50, no. 11, pp. 1736-1748, 2005.

[15] Y. Guo and B. Huang, "Moving horizon estimation for switching nonlinear systems," Automatica, vol. 49, no. 11, pp. 3270-3281, 2013.

[16] A. Alessandri, M. Baglietto, and G. Battistelli, "Min-max movinghorizon estimation for uncertain discrete-time systems," SIAM J. Control and Optimization, vol. 50, no. 3, pp. 1439-1465, 2012.

[17] L. Fagiano and C. Novara, "A combined moving horizon and direct virtual sensor approach for constrained nonlinear estimation," Automatica, vol. 49, no. 1, pp. 193-199, 2013.

[18] A. Alessandri and M. Awawdeh, "Moving-horizon estimation with guaranteed robustness for discrete-time linear systems and measurements subject to outliers," Automatica, vol. 67, pp. 85-93, 2016.

[19] Y. Wan, T. Keviczky, M. Verhaegen, and F. Gustafsson, "Data-driven robust receding horizon fault estimation," Automatica, vol. 71, pp. 210-221, 2016.

[20] S. Hanba, "Further results on the uniform observability of discretetime nonlinear systems," IEEE Trans. Automat. Control, vol. 55, no. 4, pp. 1034-1038, 2010.

[21] D. Lengani, D. Simoni, M. Ubaldi, P. Zunino, and F. Bertini, "Experimental investigation on the time-space evolution of a laminar separation bubble by proper orthogonal decomposition and dynamic mode decomposition," Journal of Turbomachinery, vol. 139, no. 3, p. 031006, 2017. 